\title{
Fabrication of single-mode channel polymer waveguides using vacuum assisted microfluidic soft lithography
}

\section{S. Baig \\ G. Jiang \\ Q. Sun}

M. R. Wang

mwang@miami.edu
The Department of Biomedical Engineering, University of Miami, Coral Gables, FL, USA

The Department of Electrical and Computer Engineering, University of Miami, Coral Gables, FL, USA

New Span Opto-Technology Inc., Miami, FL, USA

The Department of Electrical and Computer Engineering, University of Miami, Coral Gables, FL, USA

We report on the fabrication of single-mode channel waveguide structures produced by the vacuum assisted microfluidic soft lithography technique. The soft lithographic technique in conjunction with a specially designed photomask pattern and a synthesized uV curable epoxy resin can result in a high yield, cost-effective method for fabrication of channel waveguides. In particular, the use of the microfluidic technique allows for the production of freestanding high quality single-mode channel waveguides on various substrates. Notably, the introduction of sectional flow tapers allows for proper uniform filling of long length small cross-sectional waveguide structures that would ordinarily succumb to clogging at shorter distances during channel filling. The fabricated polymer waveguide was $30 \mathrm{~mm}$ in length with a cross section dimension of $7 \times 7 \mu \mathrm{m}$. The single-mode waveguide propagation was confirmed with beam profiler measurement, and the measured propagation loss for this polymer waveguide was about $0.55 \mathrm{~dB} / \mathrm{cm}$.

[DOI: http://dx.doi.org/10.2971/jeos.2013.13076]

Keywords: Integrated optics, polymer waveguides, microfluidics, soft-lithography

\section{INTRODUCTION}

Soft lithographic fabrication techniques in conjunction with ultraviolet (UV) curable resins have been used to realize high quality micro and nanostructures [1, 2]. It also provides a viable and accessible solution for fabrication of polymer optical waveguide (WG) devices [3, 4]. The use of a polydimethylsiloxane (PDMS) elastomeric stamp in microtransfer molding $(\mu \mathrm{TM})$ soft lithographic fabrication allows for a rapid replication of WG components containing both large and small cross sectional dimensions.

However, the $\mu \mathrm{TM}$ technique also adds a non-uniform $1 \sim 5 \mu \mathrm{m}$ thick planar layer that results in unwelcome variations of designed optical guiding parameters, while also potentially introducing detrimental WG cross-talks. Elimination of this planar residue layer has recently been documented by us through the use of a vacuum assisted microfluidic (VAM) technique [5, 6].

Microfluidics is a technique by which micron sized conduits are used to navigate micro and nano liter volumes of fluid through the use of valves, pumps, and/or capillary motion. VAM is a modification where a UV curable core WG resin is propelled by a vacuum suction force through a channel WG PDMS mold via inlet and outlet tunnels located at the opposite ends of the channel. The inlet tunnel acts as the reservoir whereby the resin is placed, while the outlet tunnel with a vacuum gathers the propagating fluidic resin. Thus the VAM technique allows for a controlled dynamic liquid formation of a WG channel without development of an underlying remnant layer. Once the channel is entirely filled, the WG is then solidified through UV curing. Here a specially synthesized UV curable resin plays an important role during WG fabrication.

The ability to construct single-mode (SM) polymer waveguides is a key element for the realization of polymer-based optical devices and circuitry [7]. Unfortunately, the previous VAM fabrication of WGs [5, 8, 9] has dimensional limitations. When the WG cross sectional dimension is larger than $20 \times 20 \mu \mathrm{m}$, WG lengths of over $30 \mathrm{~cm}$ can be easily fabricated. However, the viscosity of the core waveguide resin in the PDMS mold structure limits the fabrication of long length but small cross sectional dimension channel WGs. In addition the condition for visible wavelength SM WGs of a certain cross-sectional size require that the core and cladding refractive indices to differ by a tiny amount. Therefore the two main problems that must be resolved for fabricating SM WGs are listed as follows:

1. The complete filling of the narrow channel, with the UV curable resin, that will later be realized as a SM WG after it has been cured. 
2. The precise tuning of refractive index difference between the core WG relative to the surrounding cladding layer.

Usage of our previously developed low viscosity UV curable resins as well as testing of other commercially available lower viscosity UV curable resins proved ineffective for addressing the first problem. A resolution to ensure full propagation and complete filling instead concentrated on a design modification for navigating and improving flow to all sections of the waveguide. As for the second problem, the commercially available UV curable resins were not suitable as there were a limited selection with different refractive indices. More problematically the refractive indices were listed to only 2 significant figures and for SM WGs the differences between core and cladding would require the indices to be known to at least 3 significant figures. To resolve this, we focused on controlling the refractive index during the development of custom made UV curable resins for both the core and cladding layers.

We report herein our recent development of a long length, small cross sectional WG fabricated by the VAM technique through the introduction of sectional flow tapers in conjunction with the use of a synthesized low viscosity refractive index controlled UV curable resins. Notably, the refractive index of the resin can be adjusted during resin formulation, allowing for development of both the WG core and the cladding layers. As such, long length $(30 \mathrm{~mm})$ single-mode (SM) channel WGs at a $660 \mathrm{~nm}$ wavelength with cross sectional dimensions of $7 \times 7 \mu \mathrm{m}$ have been realized.

\section{DEVICE STRUCTURE AND ANALYSIS}

Based on the theory of guided wave optics, the number of modes $M$ in a rectangular waveguide referring to a square cross section of width $d$ and numerical aperture $N A$ can be approximated as $[8,10]$

$$
M \approx \frac{\pi}{4}\left(\frac{2 d}{\lambda_{0}}\right)^{2} N A^{2}
$$

where $\lambda_{0}$ is the wavelength of light in the free-space, $N A=\left(\left(n_{\text {core }}\right)^{2}-\left(n_{\text {clad }}\right)^{2}\right)^{0.5}$. M should be less than 1 for a SM WG. The relationship between waveguide cross-section width $d$ and refractive index difference between the core and cladding $\Delta n=\left(n_{\text {core }}-n_{\text {clad }}\right)$ for single mode condition can be deduced as

$$
\frac{\pi}{2}\left(\frac{2 d}{\lambda_{0}}\right)^{2} n_{\text {clad }} \Delta n<1
$$

Utilizing this equation and assuming an input wavelength of $660 \mathrm{~nm}$ and a cladding index of 1.535, the condition for a waveguide to be single mode based on the square cross sectional width and the index difference between core and cladding is shown in Figure 1 . The cladding index $\left(n_{\text {clad }}\right)$ was chosen based on our previous work of UV curable polymer resins whose refractive index fell within the 1.53-1.54 range. Referring back to the Figure 1, the region for the single mode propagation of a square waveguide was located to the left part of the curve. Based on this graph and assuming a $7 \times 7 \mu \mathrm{m}$ cross sectional dimension was desired for a SM WG at a $660 \mathrm{~nm}$ wavelength, the refractive index difference between

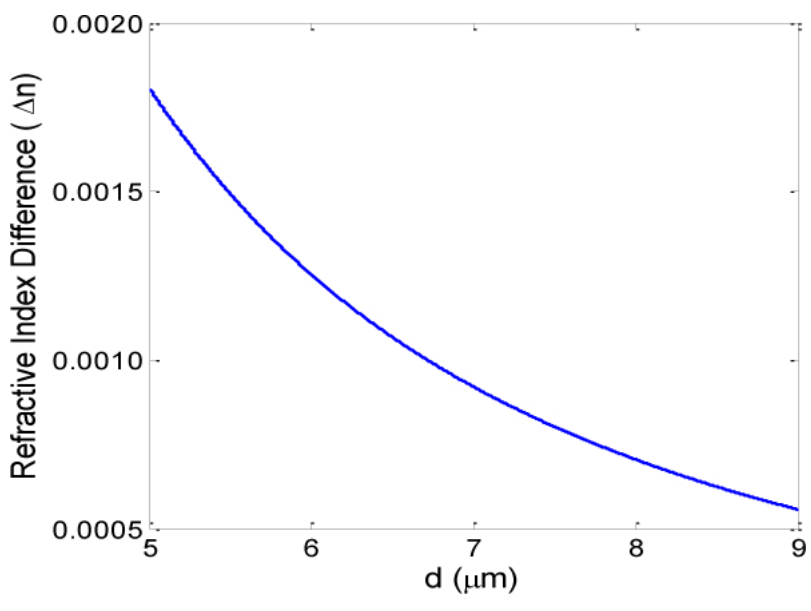

FIG. 1 Refractive index vs. Waveguide Cross-sectional width for $\mathrm{n}_{\text {clad }}=1.535$

the core and cladding would need to be approximately 0.001 $(\Delta \mathrm{n} \sim 0.001)$ for a cladding index around 1.535.

Unfortunately the development of a long length $7 \times 7 \mu \mathrm{m}$ cross sectional SM WG would be difficult as our previous VAM fabrication was only able to produce channel WGs with relatively large cross sectional dimensions. $30 \times 16 \mu \mathrm{m}$ multimode WGs and $9 \times 5 \mu \mathrm{m} \mathrm{SM} \mathrm{WGs} \mathrm{at} \mathrm{a} 1550 \mathrm{~nm}$ wavelength were previously achieved, however the SM WG produced was quite short $(\sim 2 \mathrm{~mm})$ due to the flow limitation and subsequent clogging caused by the viscosity of the resins. Therefore the production of longer length SM WGs at shorter visible wavelengths would prove to be increasingly difficult due to the viscosity related channel clogging. A significant reduction of the viscosity of the UV curable core resin would help to alleviate the channel clogging, but an investigation of commercially available UV resins as well as a reformulation of our previously developed UV resins did not yield any resins with a significantly reduced viscosity to help eliminate the clogging.

To overcome this viscosity limitation, we introduce sectional flow tapers into the microfluidic channels to help maintain proper fluid flow and prevent clogging. To implement this feature a VAM system consisting of a narrow $(7 \times 7 \mu \mathrm{m}$ cross section) flow channel (the desired small cross section SM WG channel) and two parallel surrounding wide flow channels $(150 \times 7 \mu \mathrm{m}$ cross section) was designed with perpendicular sectional flow tapers connecting the narrow channel to the wide channels. The schematic of this VAM system in Figure 2 (not drawn to scale) illustrates how the dispensed low viscosity material flows through the structure starting from the inlet hole. The presence of wide flow channels $(150 \times 7 \mu \mathrm{m})$ supports the flow of the material through the VAM structure from inlet to wide in-flow (WIF) channel, to narrow flow channel, to wide out-flow (WOF) channel, and finally to outlet. Because the material may have trouble fully propagating through the narrow flow channel even under vacuum assistance, the added sectional flow tapers can help fill the narrow channel with the material section by section. After the narrow flow channel is fully filled the material could then be confined within the channels by plugging the inlet and outlet holes or solidified, should the enclosed material have that capability (i.e. such as heating or curing of the material). 


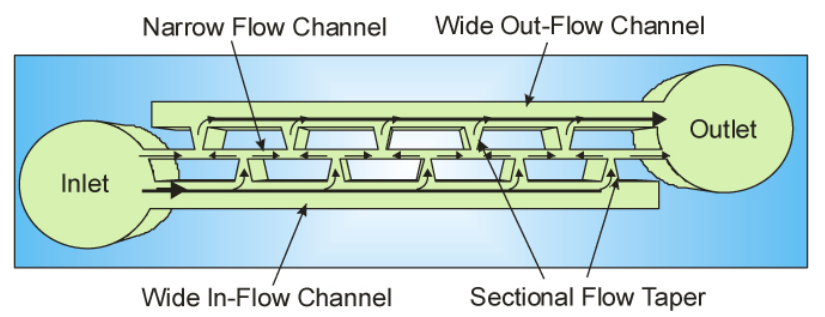

FIG. 2 Schematic of VAM fabrication using both narrow and wide channels with sectional flow tapers for the fabrication of long length small cross section channel WGs.

For our device UV curing of the dispensed UV curable polymer resins would allow for the core WG resin to solidify and thus realize the small cross sectional channel WG. Notably this would simultaneously form what may be considered as multimode WGs in the wide flow channels (with the WOF channel being partially filled). For our design the presence of these multimode WGs will have nearly no effect to the SM WGs due to the large separation $(46.5 \mu \mathrm{m})$ between the WGs.

Simulations done using BeamPROP (from Rsoft, Inc.) also confirmed that the perpendicular oriented sectional flow tapers will not introduce significant SM WG losses from the small $\sim 10 \mu \mathrm{m}$ flow taper openings that expands to $50 \mu \mathrm{m}$ before connecting to the wide flow channels. As seen in Figure 3 the inclusion of 30 perpendicular tapers alternatively spaced $1 \mathrm{~mm}$ apart from each other to the SM WG (Figure 3(a).) results in a total waveguide power loss of less than $4 \%$ (Figure $3(b)$ ) and each additional taper itself contributes to a less than $0.13 \%$ power drop per taper. The mode field diameter of this simulated waveguide at the $660 \mathrm{~nm}$ wavelength was calculated to be around $12.7 \mu \mathrm{m}$.

As a result, the improved design with the assistance of sectional flow tapers should allow for a novel VAM construction of SM WGs. Combined with the refractive index condition mentioned earlier, the desired SM WGs could then be fabricated provided that a low viscosity UV curable resin capable of satisfying the minute core/cladding index difference was synthesized. The introduction of sectional flow tapers may appear complicating the SM WG fabrication by the VAM technique. However, it is worthy to note that the presented solution would allow fabricating multi-section WG elements of different functional materials on the common substrate that would otherwise be difficult by using conventional lithography techniques.

\section{MATERIALS SYNTHESIZED}

In order to verify the device structure and analysis presented above, a new refractive index controlled low viscosity UV curable resin needs to be synthesized. The viscosity of the core WG resins plays a significant role for the realization of channel WG structures obtained through the VAM fabrication. Low viscosity core resins allow for improved laminar flow for a quicker distribution and filling of the channels, but at the potential cost of the intermittent formation of bubbles and a nonuniform density profile along both the length and the central axis of the WG. High viscosity core resins do not have these as- (a)

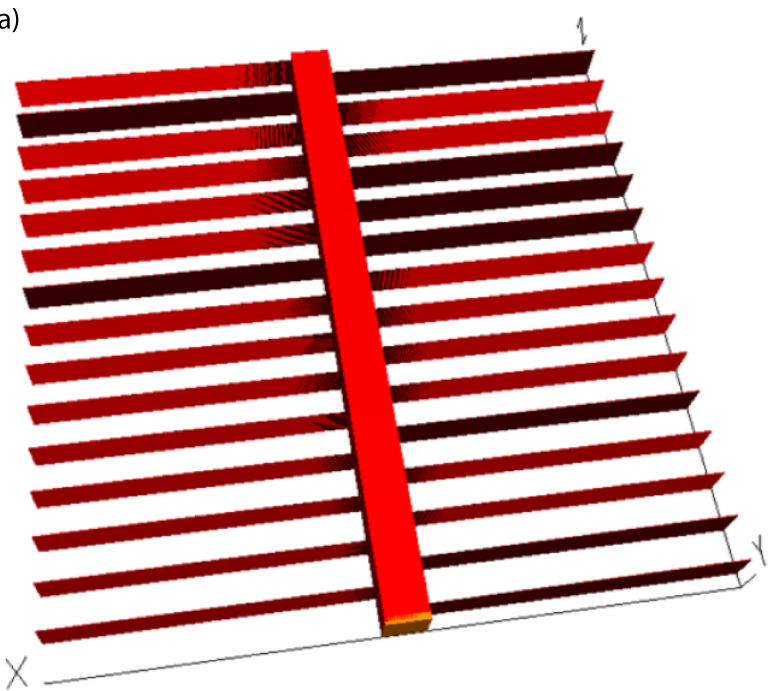

(b)
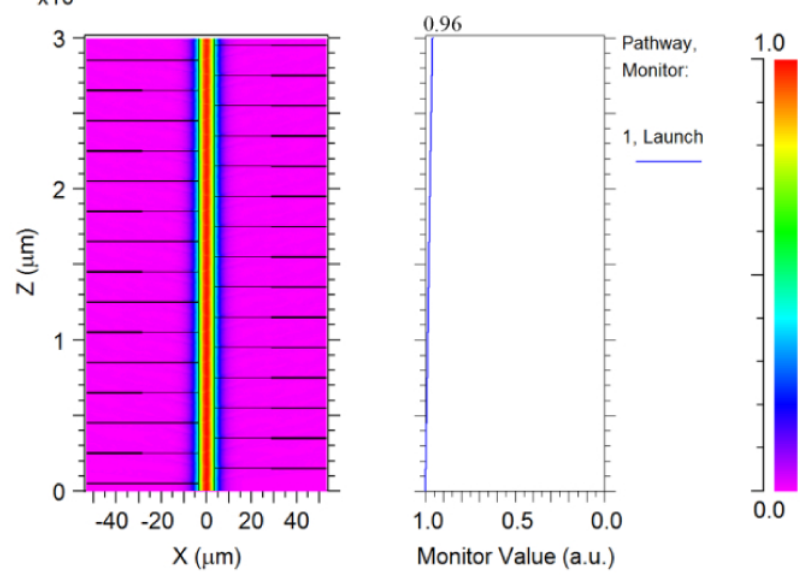

FIG. 3 BeamPROP simulation detailing the power loss attributable to sectional flow tapers.

sociated problems (or at least not as prevalent), but must contend with a slower filling rate and a higher occurrence of clogging which results in partially filled channels. Thus achieving an optimum viscosity, in addition to incorporating sectional flow tapers within the design, is necessary to ensure proper filling and ultimately the realization of high quality long length SM WGs with small cross sectional dimensions. In addition, the UV cured core WG resin must not have a strong adhesion to the PDMS mold, as removal of the mold may otherwise strip the core WG structure from its bottom cladding layer, as it remains adhered to the mold itself.

Unfortunately, based on numerous reformulations of our previously developed UV curable core resins, a significant reduction (i.e. one order of magnitude or more) of the viscosity proved difficult without adversely affecting the desired low thermal expansion, low curing time, and excellent adhesive properties that made the resin suitable in the first place. More problematically the differences in refractive index between different formulations provided no readily apparent pattern for easily increasing or decreasing the index. Instead the addition of 1,6 Hexanediol diacrylate, tech 800 to a slightly reformulated version of our previous UV curable resin recipe helped in adjusting the refractive index based on the quantity of this chemical. Notably this was achieved while not hav- 
ing any adverse effects on the rest of the resins desired features that originally made it suitable for fabricating WGs. The method for producing both core and cladding resins was then developed based on the addition of this chemical and is listed below.

1. 2,6-Di-t-butyl-4-methyl-phenol was mixed in a vial with Bis(3,4-epoxy cyclohexylmethyl) adipate for one hour.

2. Poly[(o-cresylglycidyether)-co-formaldehyde] was then added and mixed until all the crystals dissolved.

3. 3,4 Epoxycyclohexyl methyl-3,4-epoxy cyclohexane carboxylate was then added and mixed for one hour.

4. Finally, 3-glycidoxyprpyl trimethoxy silane, UVI-6992 Cyracure, and 1,6 Hexanediol diacrylate, tech 800 were added and mixed together until a homogeneous low viscosity solution was formed.

All of the above mentioned steps were done at a temperature of $65^{\circ} \mathrm{C}$, while being magnetically stirred at $300 \mathrm{RPM}$. Due to the presence of photoinitiator, the UV curable resin was kept away from light during heating and stirring and once the solution was formed it was then kept at $2{ }^{\circ} \mathrm{C}$ for storage and to prevent exposure to light. For optimal use, the solution needs to be warmed up to room temperature just prior to VAM fabrication of SM WGs. By varying the concentration of the bulk modulus component 1,6 Hexanediol diacrylate, tech 800, two different UV curable resins were synthesized and measured to have refractive indices of 1.536 for the core WG and 1.535 for the cladding layer at a $660 \mathrm{~nm}$ wavelength using an Ellipsometer by Angstrom Sun Technologies. Figure 4 shows the spectral transmittance of the UV curable core resin of $15 \mu \mathrm{m}$ thickness as it was measured using a spectrometer (Ocean Optics USB 2000) and indicates a very high transmittance of the $660 \mathrm{~nm}$ wavelength.

\section{FABRICATION AND DISCUSSION}

With the development of core and cladding UV curable resins with an index difference of 0.001 and the introduction of sectional flow tapers to the microfluidic design, a SM WG of $7 \times 7 \mu \mathrm{m}$ cross section at the $660 \mathrm{~nm}$ wavelength could then be realized. To achieve this, first a mask structure for PDMS mold fabrication was prepared by laser direct writing on a chromium glass mask. There are several sectional flow tapers connecting from the WIF channel and the WOF channel to the middle narrow flow channel where the sectional flow taper separation is $1 \mathrm{~mm}$, short enough to ensure proper VAM filling of the narrow flow channels. The mask pattern was then transferred by photolithography onto an SU-8 coated silicon wafer. Permacel tape was placed directly over a portion of the inlet and outlet holes and standard $3 \mathrm{cc}$ plastic pipette columns were then affixed normal to these taped regions to form $3 \mathrm{~mm}$ diameter inlet and $2 \mathrm{~mm}$ diameter outlet holes. Aluminum freezer tape was used to encase the wafer around its circumference, after which about $140 \mathrm{ml}$ of PDMS premixed polymer was poured within this region. After being left to settle and solidify for 24 hours at room temperature, the PDMS

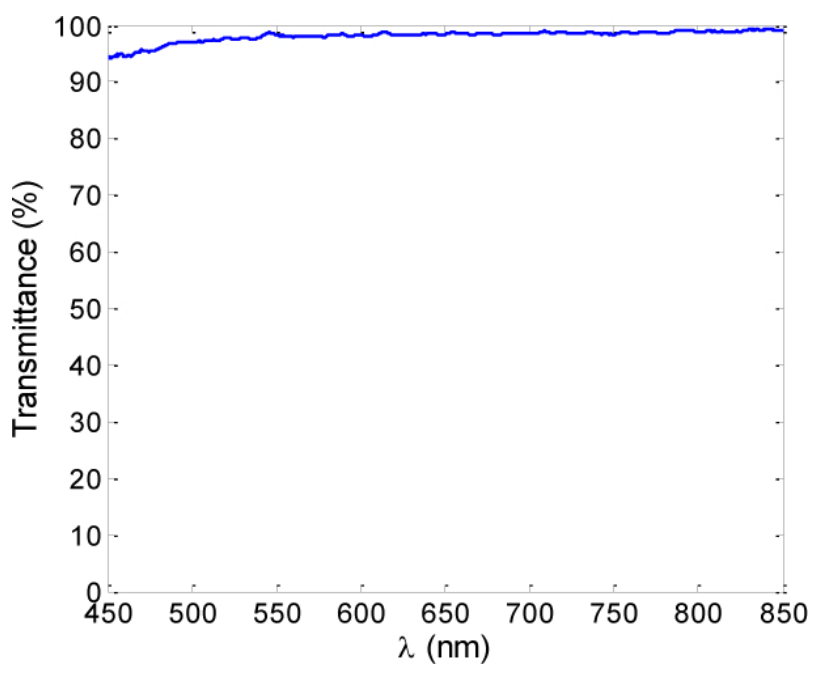

FIG. 4 Transmission properties of the synthesized UV curable core resin.

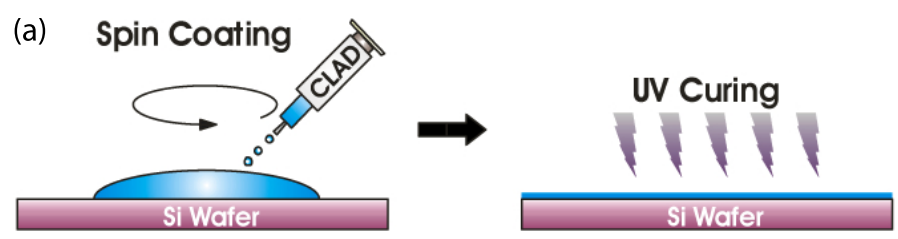

(b)
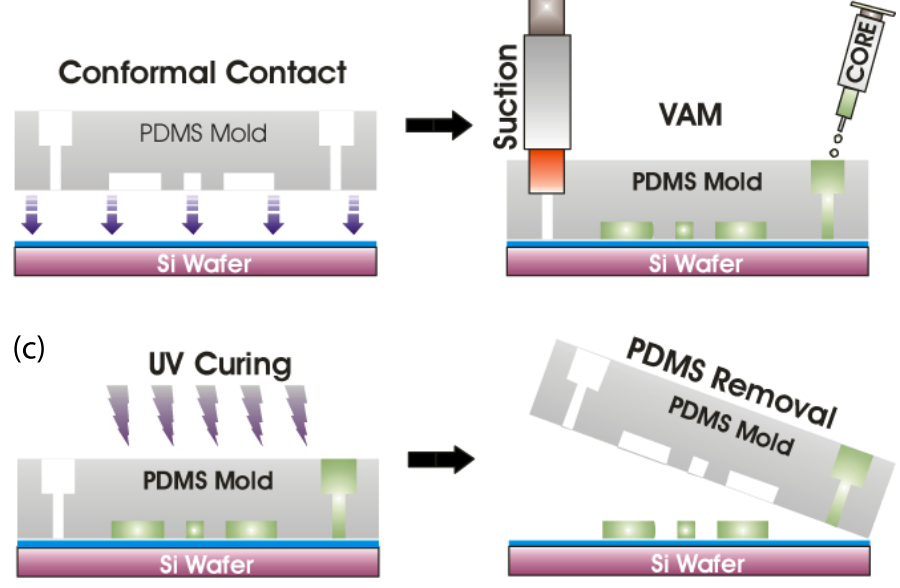

(d)
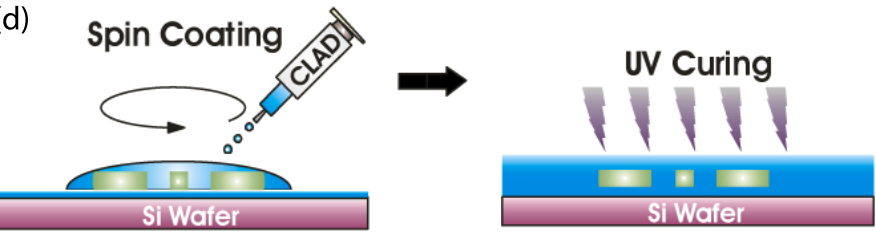

FIG. 5 Process design flow for device fabrication using VAM. (a) Spin coating and UV curing of a uniform cladding layer on a silicon wafer substrate. (b) Placing of the PDMS mold on the cladding layer and filling of the channels with the UV curable core resin by the VAM process. (c) UV curing of the filled core channels and subsequent PDMS removal to reveal the free standing channels. (d) Spin coating and UV curing of an upper cladding layer over the core channels.

stamp was now ready for use after it had been carefully peeled off from the SU-8 master.

With the prepared PDMS mold and the specially synthesized UV-curable core and cladding WG resins, long length SM WGs with small cross sectional dimension were then fabricated using the VAM technique. The fabrication process follows the procedure described earlier and depicted in Figure 5. 
A clean 4 inch diameter Si wafer was spin coated with our designed cladding material, and then uniformly UV cured by illuminating the surface area with a $25,000 \mathrm{~mW} / \mathrm{cm}^{2} \mathrm{UV}$ light source for 5 minutes to solidify the cladding layer, as shown in Figure 5(a). The PDMS mold was then placed in conformal contact with the UV cured cladding layer. A $2 \mathrm{~mm}$ diamter tube with a syringe was inserted to the outlet location of the PDMS mold. Three drops ( $<1$ cc solution) of the UV curable core WG resin were placed in the inlet reservoir (Figure 5(b)). The syringe was slowly pulled by about half an inch and fixed in position to apply the needed suction to the outlet. A CCD camera with a $30 \times$ lens magnification was positioned on top to monitor the vacuum resin flow process. The core waveguide resin flows through the WIF channels to the narrow flow channels and to the WOF channels through sectional flow tapers, and finally to the outlet holes where suction is applied. During surveillance, the formation of bubbles due to the parallel filling through different sectional flow tapers was observed, but notably migrated away from the narrow flow channel and propagated into WOF channel. The VAM resin flow process took about 45 minutes to fill up a full $30 \mathrm{~mm}$ length SM channel WG, after which the suction was removed to stop any further movement of the core WG resin. The same high intensity UV light was used to cure the core WG resin for about 5 minutes, and the PDMS mold was then gently lifted off leaving the UV hardened WG structures on top of the cladding layer, as shown in Figure 5(c). An upper cladding layer was then added by spin coating and UV curing the cladding material over the previous structures, as shown in Figure 5(d). After the VAM polymer WG fabrication, the wafer was cleaved perpendicular to the SM WG to exclude the inlet and outlet portions, thus finishing the VAM fabrication process.

Figure 6 shows the scanning electron microscope (SEM) images of the fabricated polymer WG before upper cladding layer coating. Because of the presence of WIF channels, small flow channels, and WOF channels, the fabricated device will simultaneously have the desired SM channel WG as well as remnant multimode WGs. Figure 6(a) presents a top view of a portion of the SM WG structure with the flow tapers and the surrounding fully filled WIF channel and partially filled WOF channel. Figure 6(b) shows a cross sectional view of the fabricated SM WG that clearly demonstrates the desired $7 \times 7 \mu \mathrm{m}$ cross sectional dimensions. Notably, the core WG side wall geometry is both smooth and perpendicular as it lays on the uniform planar layer consisting of the cladding material.

The fabricated SM WG with claddding layer were used for optical meausrement. A single mode fiber patch cable with a mode field diameter of $4.6 \mu \mathrm{m} @ 680$ nm (Thorlabs P1630A-FC-5) was coupled by a $660 \mathrm{~nm}$ wavelength light to one end of the cleaved WG and the output spot was observed and analyzed at the other end using a laser beam profiler (New Span Opto-Technology Beam Profiler 4.0). Figure 7(a) shows the near-field output profile captured with a $40 \times$ lens, while Figure 7(b) shows its 3D intensity distribution, confirming its single-mode 3D Gaussion distribution. In Figure 7(b) the $\mathrm{z}$-axis scale represents the intensity distribution while the $x$ and $y$ axes that have no units represent the plane of the camera sensor upon which the output profile was captured. The propagation loss of the fabricated SM WG was measured
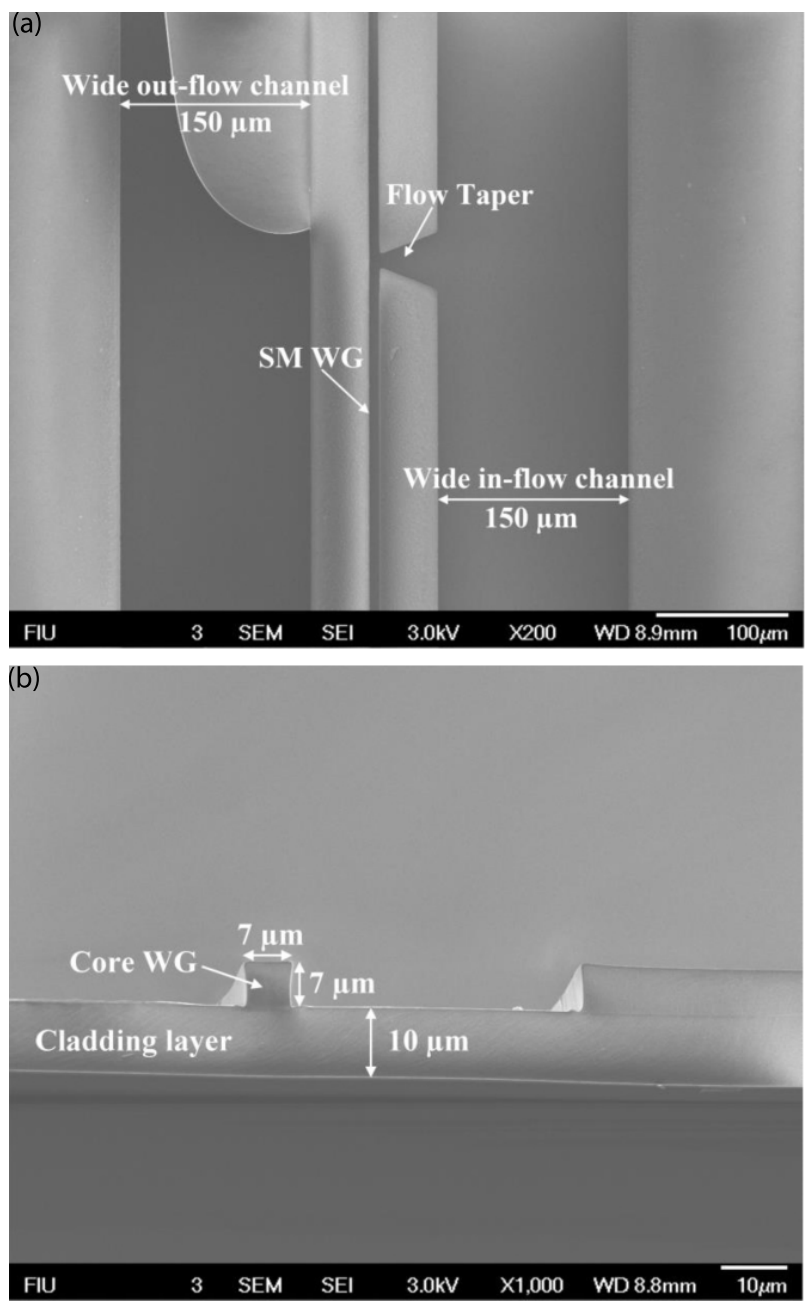

FIC. 6 SEM images of (a) top view of the SM WG (middle) with a surrounding WOF channel (left) and a WIF channel (right) and (b) cross-sectional view.

via the conventional cutback method, and was found to be $0.55 \mathrm{~dB} / \mathrm{cm}$.

\section{CONCLUSION}

In conclusion, the successful formation of long length small cross section visible wavelength SM WGs by the VAM technique has been achieved through the introduction of sectional flow tapers that prevents resin clogging during microfluidic filling that would otherwise emerge due to the reduced flow caused by the viscosity of the resins. The resulting channel WG has an excellent side wall quality and no remnant planar layer as is commonly formed by $\mu \mathrm{TM}$ fabrication. In addition, the refractive indices of the UV curable core and cladding resins have been carefully calibrated to allow for successful VAM fabrication of SM WGs. The combination of the specially formulated low viscosity refractive index controlled UV curable resins and the inclusion of sectional flow tapers helped to navigate and improve flow for cross sectional dimensions as small as $7 \times 7 \mu \mathrm{m}$. The VAM technique with sectional flow tapers allows for cost effective soft-lithographic fabrication and implementation of various types of polymer based photonic integrated circuits that require SM WGs. 

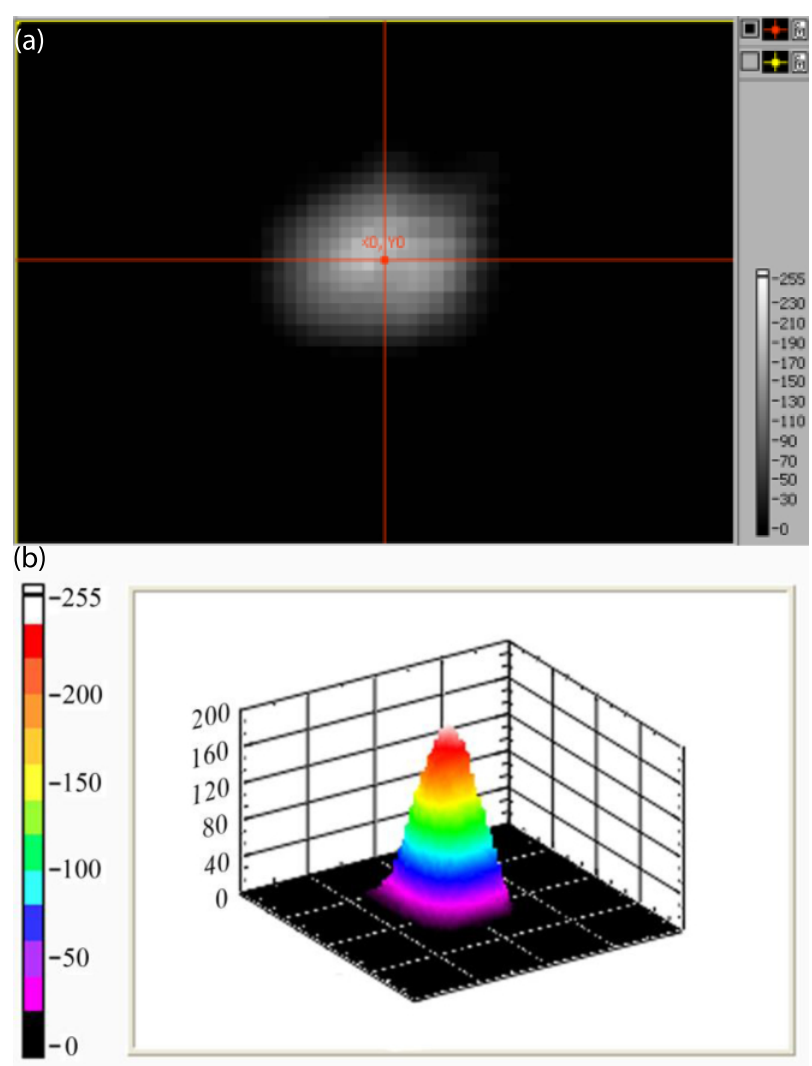

FIG. 7 The near-field output profile (a) captured with a $40 \times$ lens (with the peak intensity represented by the red crosshair) and (b) illustrated in a $3 \mathrm{D}$ intensity distribution pattern that was analyzed by a beam profiling software to confirm the $\mathrm{TEM}_{00}$ mode of the fabricated SM WG. The grey scale from 0-255 represents the intensity of the captured signal on the 8-bit camera sensor for each pixel.

\section{ACKN OWLEDGEMENTS}

This work was sponsored by the National Science Foundation. The authors would like to thank Hui Lu and Kai Shen for useful technical discussions.

\section{References}

[1] D. Losic, J. G. Mitchell, R. Lal, and N. H. Voelcker, "Rapid Fabrication of Micro- and Nanoscale Patterns by Replica Molding from Diatom Biosilica," Adv. Funct. Mater. 17, 2439-2446 (2007).

[2] P. Kim, K. W. Kwon, M. C. Park, S. H. Lee, S. M. Kim, and K. Y. Suh, "Soft Lithography for Microfluidics: a Review," Biochip J. 2, 1-11 (2008).

[3] J. Teng, H. Yan, L. Li, M. Zhao, H. Zhang, and G. Morthier, "Simple ultraviolet-based soft-lithography process for fabrication of lowloss polymerpolysilozanes-based waveguides," IET Optoelectron. 5, 265-269 (2011).

[4] T. Ishigure, and Y. Nitta, "Polymer optical waveguide with multiple graded-index cores for on-board interconnects fabricated using soft-lithography," Opt. Express 18, 14192-14201 (2010).

[5] A. Flores, S. Song, S. Baig, and M.R. Wang, "Vacuum Assisted Microfluidic Technique for Fabrication of Guided Wave Devices," IEEE Photonic. Tech. L. 20, 1246-1248 (2008).

[6] A. Fujii, T. Shimizu, K. Shimizu, K. Yatsuda, M. Igusa, S. Ohtsu, and E. Akutsu, "A novel fabrication technology of polymer optical waveguide and its application," Proc. SPIE 6775, 677501-677506 (2007).

[7] J. Scheuer, and A. Yariv, "Fabrication and characterization of lowloss polymeric waveguides and micro-resonators," J. Europ. 0pt. Soc. Rap. Public. 1, 06007 (2006).

[8] A. Flores, and M. R. Wang, Lithography (Sciyo Books, Rijeka, 2010).

[9] G. Jiang, S. Baig, and M. R. Wang, "Flexible Polymer Waveguides with Integrated Mirrors Fabricated by Soft Lithography for Optical Interconnection," J. Lightwave Technol. 31, 1835-1841 (2013).

[10] B. E. A. Saleh, and M. C. Teich, Fundamentals of Photonics (Second edition, John Wiley at Sons, Hoboken, 2007). 